\title{
Integrating Internal Standards into Disposable Capillary Electrophoresis Devices To Improve Quantification
}

\author{
Allison C. E. Bidulock, Pavel Dubský; Albert van den Berg, and Jan C. T. Eijkel* \\ MESA+ Institute for Nanotechnology and MIRA Institute for Biomedical Technology and Technical Medicine, EWI, University Of \\ Twente, P.O. Box 217, 7500 AE Enschede, The Netherlands \\ ${ }^{\ddagger}$ Department of Physical and Macromolecular Chemistry, Faculty of Science, Charles University, Hlavova 8, 12843 Prague 2, Czech \\ Republic
}

\section{Supporting Information}

\begin{abstract}
To improve point-of-care quantification using microchip capillary electrophoresis (MCE), the chip-to-chip variabilities inherent in disposable, single-use devices must be addressed. This work proposes to integrate an internal standard (ISTD) into the microchip by adding it to the background electrolyte (BGE) instead of the sample-thus eliminating the need for additional sample manipulation, microchip redesigns, and/or system expansions required for traditional ISTD usage. $\mathrm{Cs}$ and $\mathrm{Li}$ ions were added as integrated ISTDs to the BGE, and their effects on the reproducibility of $\mathrm{Na}$ quantification were explored. Results were then compared to the conclusions of our previous publication which used Cs and $\mathrm{Li}$ as traditional ISTDs. The in-

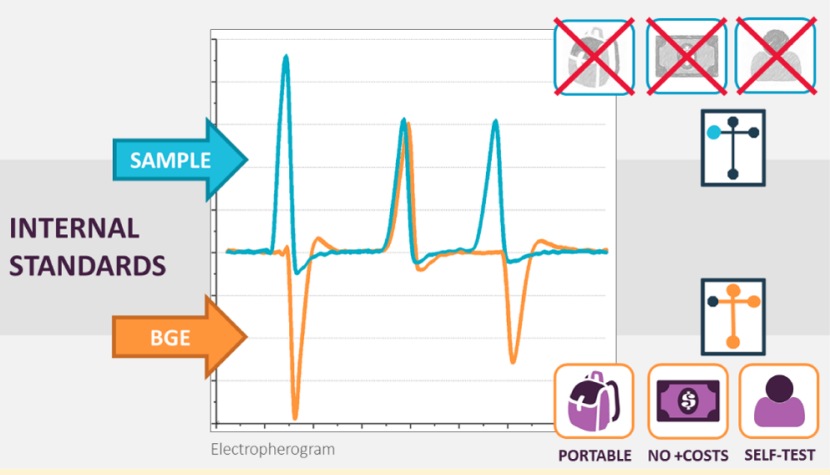
house fabricated microchips, electrophoretic protocols, and solution matrixes were kept constant, allowing the proposed method to be reliably compared to the traditional method. Using the integrated ISTDs, both Cs and $\mathrm{Li}$ improved the Na peak area reproducibility approximately 2-fold, to final RSD values of $2.2-$ $4.7 \%(n=900)$. In contrast (to previous work), Cs as a traditional ISTD resulted in final RSDs of $2.5-8.8 \%$, while the traditional Li ISTD performed poorly with RSDs of $6.3-14.2 \%$. These findings suggest integrated ISTDs are a viable method to improve the precision of disposable MCE devices-giving matched or superior results to the traditional method in this study while neither increasing system cost nor complexity.
\end{abstract}

$\mathrm{R}$ ecently, microchip capillary electrophoresis (MCE) has developed to a promising platform for point-of-care diagnosis of small ions. ${ }^{1-4}$ Adding an internal standard (ISTD) to the sample is a standard technique used to improve run-to-run quantification in conventional capillary electrophoresis $(\mathrm{CE})^{5-7}$ and MCE. ${ }^{8,9}$ In a recent paper, we demonstrated that adding an ISTD to the sample can be used not only to improve run-to-run quantification, but also improve chip-to-chip precision. ${ }^{10}$ The latter is crucial when disposable, one-use microchips are used-as is common in point-of-care (POC) analysis. However, adding an ISTD to the sample in POC analysis would require an extra sample preparation step performed by either an unskilled operator or the device itself. Integrating a microfluidic mixing system into the CE microchip would introduce new error sources, increase system complexity, and raise fabrication costs. Requiring an unskilled operator to mix the ISTD with the sample would similarly introduce new error sources and increase test complexity. These are severe limitations for a quantitative, disposable microchip.

Instead of mixing with the sample, the approach we take in this work is to add an internal standard to the background electrolyte (BGE). It is well-known from literature that for each additional ionic constituent added to the BGE, an additional system peak will also be present. ${ }^{11-13}$ Although this phenomenon is basic knowledge within the field, the literature does not report on the use of system peaks for the purposes of improving quantitative measurements. The authors of this study speculate that this is due to the complex nature of system peaks and the fact that traditional ISTDs are readily available for use by a skilled technician in a standard laboratory setting. It is only when we try to address the problem of precise measurements in disposable microchips used by unskilled operators that the potential of using a reference system peak instead of a traditional ISTD becomes attractive: no additional sample manipulation, microchip changes, or system expansions are required with this method-which makes it an ideal solution for POC applications.

Here, we present a novel method to improve chip-to-chip precision in disposable MCE devices whereby the ISTD-like component (further referred to as the integrated ISTD ion) is

Received: October 25, 2016

Accepted: February 2, 2017

Published: February 2, 2017 
added to the BGE instead of to the sample. The resulting system peak area/height from the integrated ISTD ion is then used to correct for different sources of system error in the quantitative measurement, similar to a traditional ISTD peak. We explore the effects of two integrated ISTDs (Cs and $\mathrm{Li}$ ions) on the chip-to-chip reproducibility of $\mathrm{Na}$ quantification, using a data set of 900 electropherograms collected from measuring five concentrations of $\mathrm{NaCl}$ repeatedly on six microchips. The in-house fabricated microchips, CE protocols, and reagents were the same as presented in our previous publication, ${ }^{10}$ allowing us to draw reliable conclusions about the viability of the new method. We thus compare traditional ISTDs to integrated ISTDs.

\section{EXPERIMENTAL SECTION}

Microchip CE System. This experiment set used the same chip holders, electrophoretic methods, and equipment as in previous work, to which the reader is referred for details. ${ }^{10}$

In brief, Borofloat glass CE chips were fabricated in-house using the same principles as presented earlier. ${ }^{14}$ Channels $6 \mu \mathrm{m}$ deep by $52 \mu \mathrm{m}$ wide were etched into the top glass plate, and $140 \mathrm{~nm}$ recessed platinum electrodes were sputtered onto the bottom plate. The electrodes were placed at the end of the separation channel, in contact with the fluid, and $2 \mathrm{~cm}$ away from the $200 \mu \mathrm{m}$ double- $\mathrm{T}$ intersection. After bonding and dicing, each chip is $15 \mathrm{~mm} \times 30 \mathrm{~mm} \times 1.6 \mathrm{~mm}$.

A three-piece Delrin holder extended the powder-blasted reservoirs from $4 \mu \mathrm{L}$ to $\sim 60 \mu \mathrm{L}$ and suspended four platinum wires in the wells which were connected to the high-voltage supply (CU 411, IBIS Technologies, Hengelo, The Netherlands). Two spring-loaded pins further connected the thin-film conductivity electrodes to the in-house built detector electronics. For high-voltage protocols and further electronics details, please see previous work. ${ }^{10}$

Reagents. Stock solutions of $250 \mathrm{mM}$ MES hydrate and $250 \mathrm{mM}$ L-histidine (His), 0.05\% (w/v) (hydroxypropyl)methylcellulose (HPMC), $200 \mathrm{mM} \mathrm{KCl}, 100 \mathrm{mM} \mathrm{CsCl}$, and $100 \mathrm{mM} \mathrm{LiCl}$ were prepared and stored in the refrigerator. The MES-His stock was verified to have a $\mathrm{pH}$ of $\sim 6.1$ at room temperature after mixing. Aliquots were used to prepare daily solutions of BGE: $100 \mathrm{mM}$ MES-His ( $\mathrm{pH}$ 6.1), 0.01\% (w/v) HPMC, $0.25 \mathrm{mM} \mathrm{KCl}$, and $7.5 \mathrm{mM}$ of each added reference standard $(\mathrm{CsCl}$ and $\mathrm{LiCl})$. This is the same $\mathrm{BGE}$ as presented earlier ${ }^{10}$ - sans the ISTD ions. Sample solutions were also prepared from stock daily and contained $100 \mathrm{mM}$ MES-His, $0.01 \% \mathrm{HPMC}$, and one of five different concentrations of $\mathrm{NaCl}$ $(2,4,6,8$, and $10 \mathrm{mM}$; stock was $500 \mathrm{mM})$. All chemicals were purchased from Sigma-Aldrich (Steinheim, Germany), aside from the $\mathrm{LiCl}$ stock solution which was graciously donated by Medimate (Enschede, The Netherlands). Reagents were 99\% grade or higher, and Milli-Q water was used to mix all solutions.

Experimental Procedure. Six microfluidic chips were analyzed thoroughly in our previous work, and the same six microchips were used here. Between publications, each microchip was cleaned by flowing $0.1 \mathrm{~N} \mathrm{NaOH}$ and Milli-Q water through the chip for 10 and $15 \mathrm{~min}$, respectively. The channels were then filled with BGE solution and left for at least 90 min so that the surface chemistry of the glass could adjust to the solution. The microchips were then flushed with, and stored in, Milli-Q water.

One chip "load" consists of five electrophoretic "runs", or injection-separation pairs, performed on the same aliquots of $\mathrm{BGE}$ and sample solution applied to a microchip. Each $\mathrm{NaCl}$ analyte concentration (total, 5) was loaded six times onto each microchip (total, 6); thus, 900 electropherograms were collected for this data set.

Between chip loads, all reservoirs were rinsed 3 times with Milli-Q water. The buffer waste reservoir was then rinsed 3 times and filled with BGE. This aliquot was then pulled into the microchip, displacing the used BGE solution, via vacuum pressure applied to the other three reservoirs. After $10 \mathrm{~min}$ the liquid was removed, and the remaining three reservoirs were rinsed 3 times with BGE. Amounts of $55 \mu \mathrm{L}$ of BGE and sample were then applied to their respective reservoirs immediately before running a new experiment.

Baseline Fit. Previously, ${ }^{10}$ the collected signal's baseline was approximated using a moving median subtraction of 175 points (or $0.734 \mathrm{~s}$ at a data collection rate of $238.42 \mathrm{~Hz}$ ). This estimation was sufficient as all peaks of interest in the electropherogram were positive and the baseline had minimal curvature. Here, the electropherogram contains both negative (integrated ISTD) and positive (analyte) peaks. The moving median subtraction was found to have inadequate performance in estimating the baseline, particularly across the integrated ISTD peaks, and negatively impacted the repeatability of the measurement.

Thus, this work uses a background correction (backcor) method as found on MATLAB's File Exchange and described in literature by Mazet et al. ${ }^{15}$ In short, the script applies a polynomial fit to the background using one of four predefined functions, a user-defined order, and a threshold setting which limits the influence of the peaks on the estimation. A symmetric Huber function with a polynomial order of 5 and a threshold of 0.01 was used here, which fits well to the baseline (Figure S1). Peak areas were then estimated using the full width at halfmaximum (FWHM), and the migration time was taken as the half-maximum time on the rising edge of the peak. Additional methods for calculating the peak area were investigated, including integrating the area under the peak and using the peak arrival time (i.e., mobility) as a correction factor. The FWHM approximation gave the best reproducibility.

\section{RESULTS AND DISCUSSION}

Understanding Integrated ISTD Peaks. In a basic capillary electrophoresis separation, one can think of the channel containing BGE and zones of different analyte ions moving at different mobilities toward a detector. Additional zones consisting of only BGE constituents can also appear with specific mobilities, and may deform analyte zones migrating in their vicinity. As they are a function of the BGE system itself, these regions are often referred to as "system zones" or "eigenzones". ${ }^{16}$ Mathematical models and/or simulation programs, such as PeakMaster ${ }^{17}$ and Simul 5, ${ }^{18}$ are often used to predict the mobilities and amplitudes of system peaks.

Integrated ISTD peaks are denoted in this paper with quotations because they are not moving analyte zones of Cs or $\mathrm{Li}$ ions, but rather the inverse: they are depleted regions in the BGE where notably less $\mathrm{Cs}$ and $\mathrm{Li}$ ions are present. Figure 1, illustrated using Simul 5, ${ }^{19}$ shows this phenomenon. When the sample is electrokinetically injected (EKI) into the microchip injection channel, the sample (de)stacks as it crosses the stationary boundary into the BGE. Prior to separation, the sample plug does not contain any $\mathrm{Cs}$ or Li ions-they are completely displaced by the sample zone during EKI. This discontinuity in the BGE (the sample plug) gives rise to two depleted-ion system zones moving through the separation 


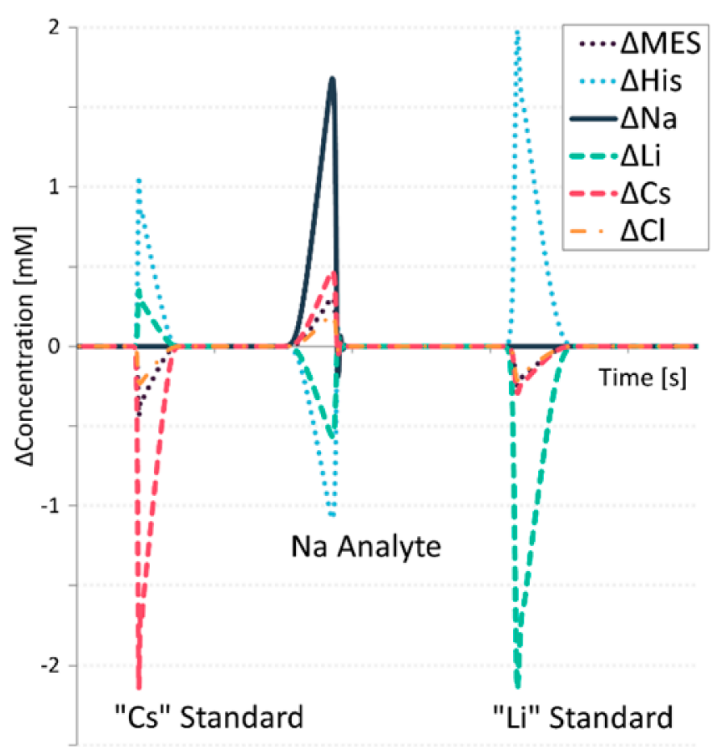

Figure 1. Ionic concentration changes between moving zones (peaks) passing the detector and the BGE, simulated using Simul 5 (ref 19). Cs and $\mathrm{Li}$ concentrations change in all moving zones; however, their corresponding system peaks constitute the most significant change for that ion.

channel with a migration velocity similar to that of the depleted ion.

However, it should be noted that, since these are BGE constituents (not analytes), these zones are not the only depleted regions for that ion in the system: rather, they are the most significantly depleted. This aspect differs from sample peaks, where each ion has only one analyte region-though in this region the concentrations of the BGE ions are also changed.

Integrated ISTDs: Mobility. Considering a BGE with multiple co-ions $\mathrm{A}$ and $\mathrm{C}$, and counterion $\mathrm{B}$, the system peak due to the addition of co-ion $\mathrm{C}$ can be derived from eq 40 of Štědrý et al. ${ }^{20}$ to have a mobility $\lambda_{\mathrm{C}}$ of

$$
\begin{aligned}
\lambda_{\mathrm{C}}= & {\left[2 c_{\mathrm{A}} c_{\mathrm{B}}\left(\mu_{\mathrm{A}}+\mu_{\mathrm{B}}\right) \mu_{\mathrm{C}}+c_{\mathrm{A}} c_{\mathrm{C}}\left(\mu_{\mathrm{A}}-\mu_{\mathrm{C}}\right) \mu_{\mathrm{B}}+c_{\mathrm{B}} c_{\mathrm{C}}\left(\mu_{\mathrm{B}}+\mu_{\mathrm{C}}\right) \mu_{\mathrm{A}}\right] / } \\
& {\left[\left(\mu_{\mathrm{A}} c_{\mathrm{A}}+\mu_{\mathrm{B}} c_{\mathrm{B}}+2 \mu_{\mathrm{C} c_{\mathrm{C}}}\right)\left(c_{\mathrm{A}}+c_{\mathrm{B}}\right)\right] }
\end{aligned}
$$

where $c$ is the analytical concentration and $\mu$ is the ion's mobility. This equation assumes that the influence of $\mathrm{H}^{+}$and $\mathrm{OH}^{-}$can be neglected, buffering ions $\mathrm{A}$ and $\mathrm{B}$ are half-ionized, and co-ion $\mathrm{C}$ is entirely ionized-which is the case of the 100 mM MES-His buffer and Cs or Li used as the integrated ISTD. With these values, Stědrýs eq 1 estimates the mobilities of the "Cs" and "Li" peaks to be $8 \%$ smaller than if the ISTDs were added to the sample. With more complicated BGEs, eq 1 may not apply; however, the mobility of the integrated ISTD-related system peak is easily predictable using special software. ${ }^{17}$ In any case, the mobility of the system peak invoked by the presence of the integrated ISTD is a well-defined quantity.

Integrated ISTDs: Peak Size. While the mobilities of system peaks (including the added standards) are dependent on the BGE composition, the size of these peaks is strongly dependent on both the BGE and the sample matrix. ${ }^{21}$ Figure 2A plots five electropherograms collected from the same chip with integrated ISTDs, each measuring a different $\mathrm{Na}$ concentration. As the concentration of $\mathrm{Na}^{+}$increases in the sample-also increasing the sample's conductivity and ionic strength—the "Cs" system peak height becomes more negative
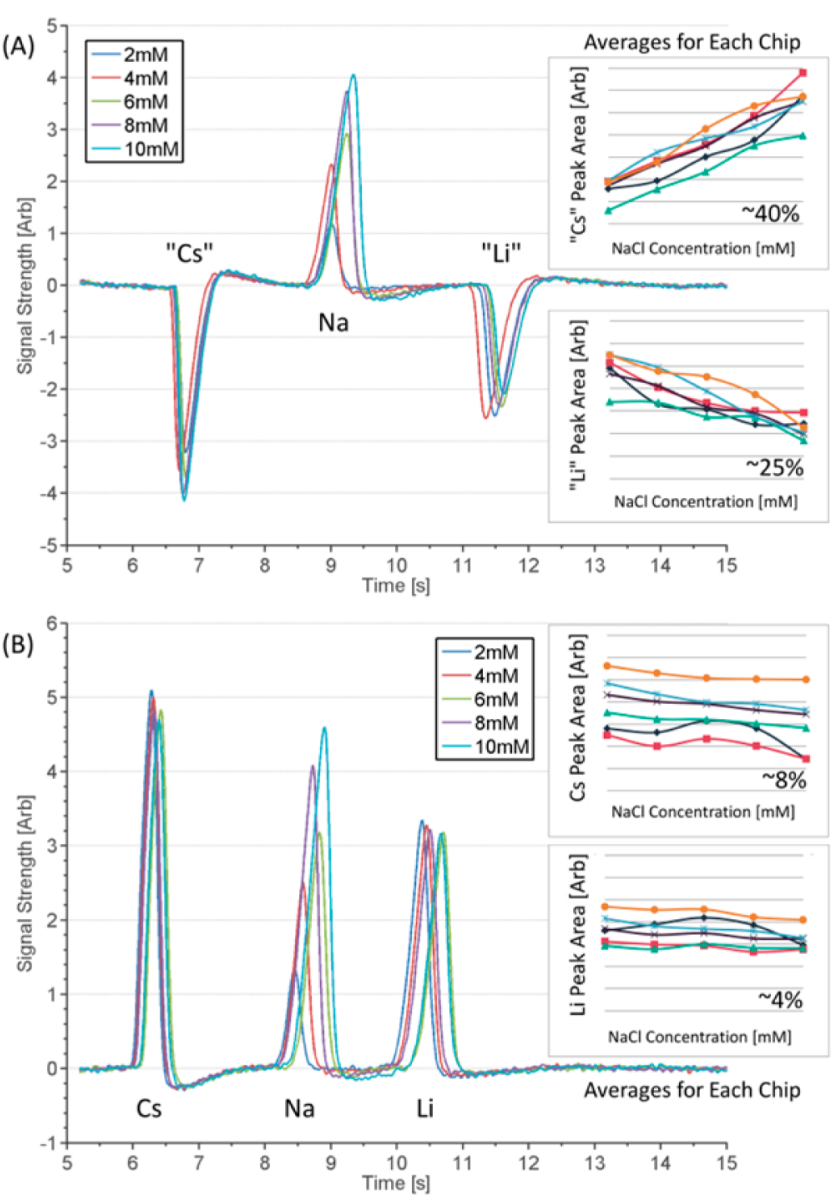

Figure 2. Cs and Li reference peaks change in area with analyte $(\mathrm{Na})$ concentration, both when added to the BGE (A) and when added to the sample (B). In both figures, five electropherograms collected from the same microchip are shown, each measuring a different $\mathrm{NaCl}$ concentration. Embedded graphs plot the average normalized areas for the reference peaks on each microchip, at each $\mathrm{NaCl}$ concentration, illustrating a general downward/upward trend. The rate of change is much more pronounced when $\mathrm{Cs}$ and $\mathrm{Li}$ are added to the BGE, as expected.

and the "Li" system peak less. With sample and BGE matrixes used here, the average size change within a single chip is approximately $40 \%$ for "Cs" and $25 \%$ for "Li" per 5 times increase in $\mathrm{NaCl}$ concentration.

This behavior conflicts with the idea of a true internal standard: a substance which is different from the analyte of interest, but is influenced by the sample preparation and measurement system in the same way. It must be noted, however, that a perfect internal standard (according to this definition) cannot exist in MCE devices which use EKI methods. Ideally, the relative amount of injected analyte is fixed and independent of time, provided that the sample is injected long enough and is restricted from diffusing into the separation channel. ${ }^{22}$ Practically, a mobility bias still exists. While the injected ions are assumed to have the same concentration ratio with respect to each other, other constituents and properties of the sample will influence the accuracy of that ratio. ${ }^{23}$ Pinching the sample in the injection phase, while preventing diffusion, can also lead to stacking of ions with faster mobilities in the sample plug. ${ }^{24}$ Figure $2 \mathrm{~B}$ similarly plots five electropherograms collected from the same chip with traditional ISTDs and shows that a slight decrease in the internal standard peak height is 
visible as the $\mathrm{NaCl}$ concentration increases. The average change within a single chip is approximately $8 \%$ for Cs and $4 \%$ for $\mathrm{Li}$ per 5 times increase in $\mathrm{NaCl}$ concentration.

In spite of these sources of variability, our previous work ${ }^{10}$ clearly demonstrated that traditional ISTDs are a means to improve precision in MCE. Thus, it is also of interest to determine if integrated ISTDs can effectively improve the precision of the measurement, despite their complex relationship with the sample and BGE matrixes. On the basis of our knowledge of system peaks, injection variation due to changes in sample properties would not expect to be accounted for by the integrated ISTD approach. For example, a weak electrolyte ISTD in the BGE would not correct for sample $\mathrm{pH}$ differences around a weak analyte's $\mathrm{p} K_{\mathrm{a}}$. Injection variation due to changes in BGE properties, channel dimensions, and variations in sample plug formation would expect to be accounted for. The next section investigates this, using simulations.

Simulations: Microchip Channel Variances. Disposable microchips suffer from variabilities that $\mathrm{CE}$ and MCE laboratory systems do not since each chip differs and each chip is only used once. Slight variance in channel depths and/or widths due to small fabrication differences will result in different sample plug volumes, leading to quantification errors. Thus, it is important to know if integrated ISTDs can account for these variations on a theoretical level.

To investigate this, Simul 5 was used to simulate the same injected sample with different plug widths (ignoring pinching effects). The double-T intersection has a length of $0.2 \mathrm{~mm}$, and six other widths were analyzed between 0.15 and $0.25 \mathrm{~mm}$. As expected, the $\mathrm{Na}$ analyte peak area increases with sample plug width: the more sample present, the more $\mathrm{Na}$ ions are also present (Figure 3A). Likewise, the integrated ISTDs also
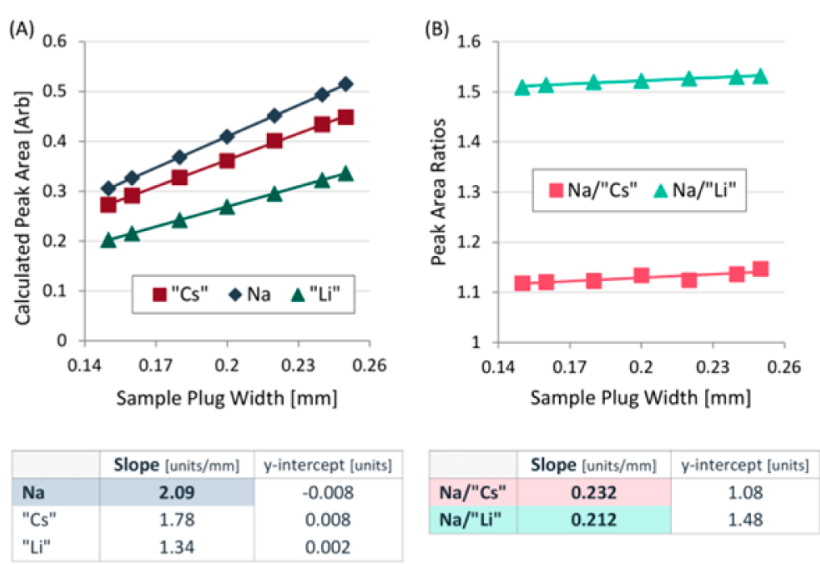

Figure 3. Variations in microchip channel dimensions were simulated by varying the sample plug width in Simul 5 . As expected, the Na analyte peak area increases with sample plug width (A), which would lead to quantification errors in disposable microchips. The peak area ratios (PARs) of $\mathrm{Na}$ with both "Cs" and "Li", however, remain constant within $10 \%$ (B) - thus indicating the BGE reference peaks would be useful correction factors.

increase in peak area with plug width: the more BGE that is displaced, the larger the moving zones of missing $\mathrm{Cs} / \mathrm{Li}$ ions are. The rate of change for each peak, however, is different: slopes $m$ are $\mathrm{Na}=2.09$, “Cs" $=1.78$, and "Li" $=1.34$ [area/ $\mathrm{mm}$ ].

With an ideal internal standard, the rate of change would be equal for both analyte and ISTD peaks, leading to a slope of zero at all plug widths when the peak area ratio (PAR) is taken. Due to the varying slopes, the PARs in these simulations are actually rational functions; however, they can be approximated as linear lines (Figure 3B) with slopes $m$ of $\mathrm{Na} /$ "Cs" $=0.232$ and $\mathrm{Na} /$ "Li" $=0.212[\mathrm{ratio} / \mathrm{mm}]$. These slopes are approximately $10 \%$ of the analyte's $(\mathrm{Na})$; in other words, while not immune to channel variances, the integrated ISTDs will minimize these influences down to $1 / 10$ th that of the analyte alone. Thus, integrated ISTDs can be used in good approximation to correct for changes in analyte peak area due to disposable chip differences.

Experiments: Migration Time Reproducibility. Relative standard deviations (RSDs) were larger between chips than within a single chip, as expected due to the chip variations mentioned. Chip-to-chip values were $2.93 \%, 2.34 \%$, and 3.69\% for $\mathrm{Na}$, "Cs", and "Li", respectively. Imprecision in chip-to-chip RSDs again increased with migration time, indicating (a) variation in separation path lengths due to varying conductivity electrode alignment and/or (b) variation in chip-to-chip electroosmotic flow (EOF) due to differences in chip surface chemistry. Intrachip and chip-to-chip RSDs are provided in Table S1.

Using the integrated ISTDs, the reproducibility of the analyte's migration time can be equally improved with both references when using a time ratio: $1.06 \%$ for $\mathrm{Na} /$ “Cs" and $0.98 \%$ for $\mathrm{Na}$ /“Li”. When adjusting the migration time of $\mathrm{Na}$ using the relative distance between both ISTDs, using the same equation from previous work, ${ }^{10}$ the RSD was further improved to $0.71 \%$, as illustrated in Figure $4 . t_{\mathrm{Cs}, \mathrm{av}}$ and $t_{\mathrm{Li} \text {,av }}$ are the average arrival times of "Cs" and "Li" over all measurements $(n=900)$, respectively.

$$
t_{\mathrm{Na}, \text { rel }}=\left[t_{\mathrm{Cs}, \mathrm{av}}\left(t_{\mathrm{Li}}-t_{\mathrm{Na}}\right)+t_{\mathrm{Li}, \mathrm{av}}\left(t_{\mathrm{Na}}-t_{\mathrm{Cs}}\right)\right] /\left(t_{\mathrm{Li}}-t_{\mathrm{Cs}}\right)
$$

Experiments: Analyte Calibration Curve Fitting. As seen previously in Figure 2A, the peak areas of "Cs" and "Li" vary with $\mathrm{Na}$ concentration differences; thus, it is useful to understand which regression type is theoretically best-suited to fit the BGE-added reference calibration curves. Electrokinetic

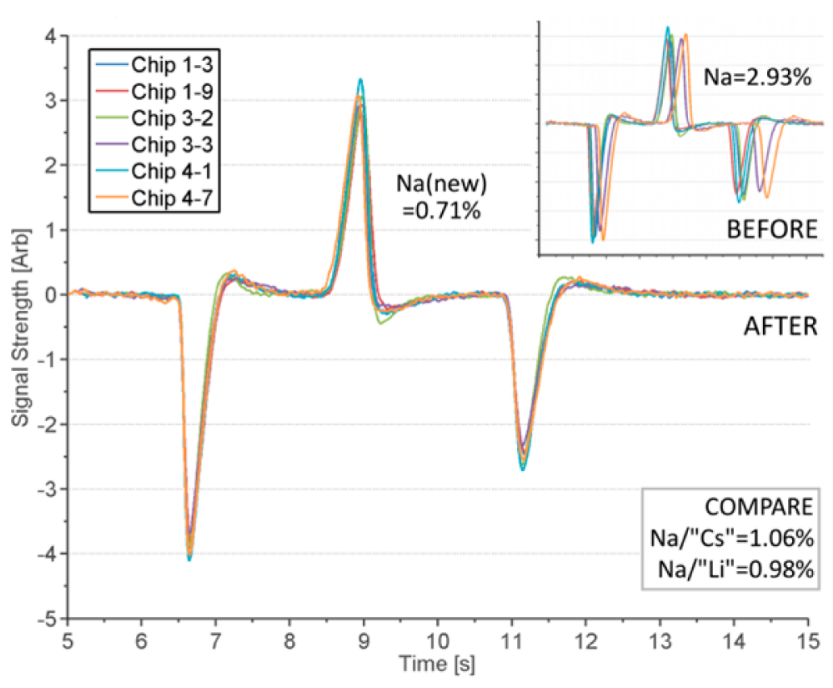

Figure 4. One electropherogram from each microfluidic chip illustrating the variations in peak arrival time (before) and the resulting correction using eq 2 (after). RSDs are given for the analyte peak and the reference peak time ratios to compare. 
injection and separation steps for multiple $\mathrm{NaCl}$ sample concentrations were investigated using Simul 5. Figure S2 plots the $\mathrm{NaCl}$ concentration in the sample well versus the simulated $\mathrm{Na}$ ion concentration in the injection channel after sufficient time has passed to allow for all sample ions to cross the stationary boundary. The simulations suggest that the EKI results in a slightly quadratic curve between 1 and $20 \mathrm{mM}$, which will then be reflected in the separated analyte peaks. Despite this quadratic dependence on the $\mathrm{Na}$ concentration in the sample well, the separated peak areas can be approximated with high confidence using a linear regression between 1 and 10 $\mathrm{mM} \mathrm{NaCl}$ (Figure 5A). The separated peak heights, however,
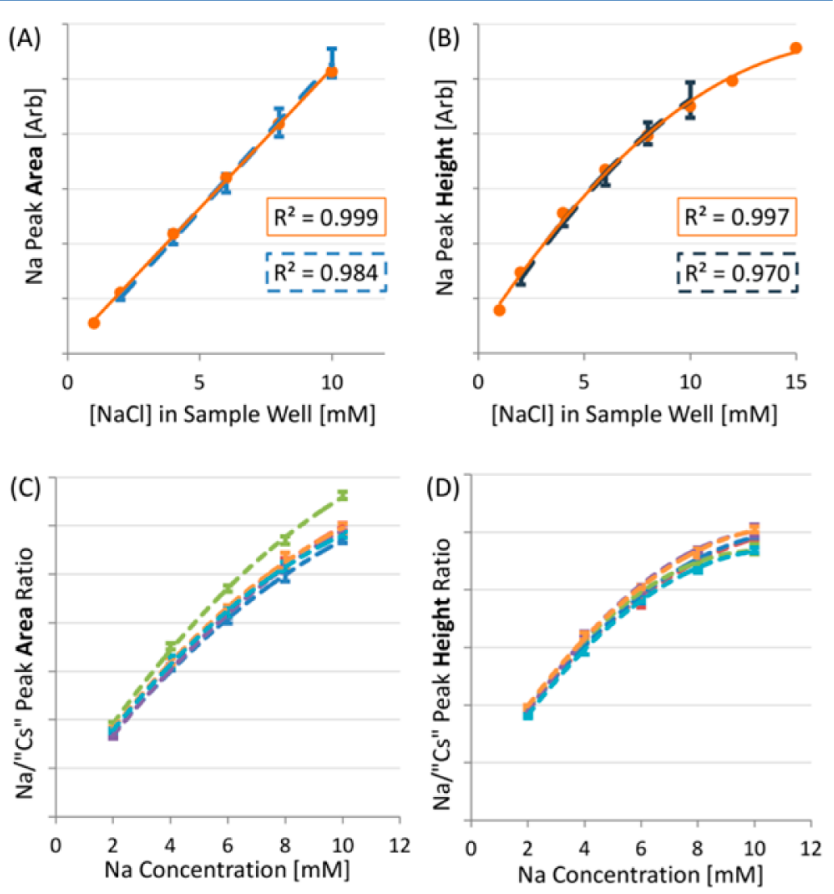

Figure 5. (A) Measurements (error bars) and simulations (points) of $\mathrm{Na}$ peak area vs the $\mathrm{NaCl}$ sample concentration, showing excellent prediction; panel $\mathrm{B}$ displays $\mathrm{Na}$ peak height. (C) Six intrachip calibration curves using the $\mathrm{Na} /$ "Cs" peak area ratios, illustrating one outlier chip; panel D shows $\mathrm{Na} /$ "Cs" peak height ratios, illustrating that the outlier chip behaves normally.

cannot (Figure 5B). Both plots compare the $\mathrm{Na}$ analyte peaks collected from Simul 5 (circles) with the experimental data collected from all microfluidic chips (error bars). Theoretical calibration lines have been scaled by a fixed constant to match the arbitrary values on the $y$-axis and are otherwise not manipulated; thus, theoretical curve shapes are in excellent agreement with the experimental results. The correlation coefficients $\left(R^{2}\right)$ for the chip-to-chip $\mathrm{Na}$ calibration curve were $0.984(x)$ and $0.970\left(x^{2}\right)$ when using peak areas and heights, respectively.

When the integrated ISTD is used for correcting the Na peak height/area, not only the EKI contributes to the nonlinearity of the calibration curve, but so does the dependency of the integrated ISTD peak size on the injected amount of analyte. Then the calibration curve is of an empirical nature whichsimilarly to the simple $\mathrm{Na}$ peak area-can be approximated by a second-order polynomial in the concentration ranges inspected in this study. When the integrated "Li" peak is used as a reference standard, the correlation coefficients improve to 0.997 (areas) and 0.988 (heights). Using the integrated "Cs" reference peak results in calibration curves with coefficients of 0.979 (areas) and 0.986 (heights). Note that the Na/“Cs” peak height correlation is comparable to the $\mathrm{Na} /$ "Li" curve; however, the peak area curve is not. When individual chip $\mathrm{Na} /$ "Cs" area calibration curves are plotted separately, one microfluidic chip is distinctly different from the others (Figure 5C). This was confirmed by performing the Dixon's $Q$ test, which identified chip 3-2 as an outlier with a $90 \%$ confidence at every concentration level, except for the lowest $(2 \mathrm{mM})$. If this chip's data is removed from the experiment set $(n=5$ not 6$)$, the $\mathrm{Na}$ / "Cs" area coefficient improves to 0.993 -comparable to "Li". It is unknown why this single chip shows significantly different behavior for $\mathrm{Na}$ /“Cs" peak areas as compared to other chips, while the $\mathrm{Na}$ /“Cs" peak heights (Figure $5 \mathrm{D}, n=6$ ) as well as the "Li" data (not shown) are seemingly unperturbed on the very same chip.

Calibration curve data for both individual and all microchips are summarized in Table S2.

Experiments: Peak Area Reproducibility. Chip-to-chip peak area and height RSDs for each $\mathrm{NaCl}$ sample concentration $(2,4,6,8,10 \mathrm{mM})$ were tested on six randomized chips according to the OECD requirements on analytical method validations. $^{25}$ Intrachip RSDs are all between $\sim 5$ and $7 \%$ without using an ISTD for correction. Chip-to-chip variability is 1.5-2 times the intrachip variability-much better than in our previous work, ${ }^{10}$ where chip-to-chip variability increased by 3-4 times. Figure S3 compares peak area RSDs at each $\mathrm{NaCl}$ concentration, for each microfluidic chip and all chips.

In the next steps, RSD values obtained with the integrated ISTD method are compared to values where either no ISTD or the traditional ISTD method is used. We notice that a statistical test for comparing RSDs values with a defined statistical significance is not readily available, and comparing mere SD values runs up against scaling problems. Nevertheless, the visual data mining method (Figure 6) enables us to make conclusions convincing enough for this proof-of-concept study.

Peak area and height ratios with both "Cs" and "Li" integrated ISTDs improve the chip-to-chip RSDs to $~ 3-5 \%$. When considering peak area ratios (Figure 6A), the "Li" integrated ISTD gives better RSDs than "Cs" at every $\mathrm{NaCl}$ concentration due to the significantly different $\mathrm{Na}$ /“Cs" values for one chip (as discussed earlier). If only data from the other five chips are considered, the $\mathrm{Na}$ / "Cs" RSD values are comparable to $\mathrm{Na}$ /“Li”. The peak height ratios (Figure 6B) show good improvement for both "Cs" and "Li" ISTDs.

As an additional means to judge how well each integrated ISTD performed, experimental correlation coefficients $(\rho)$ for the Na peak with "Cs" and "Li" were calculated with the same equation from previous work ${ }^{10}$

$$
\rho=\left(\mathrm{RSD}_{\mathrm{A}}^{2}+\mathrm{RSD}_{\mathrm{ISTD}}{ }^{2}-\mathrm{RSD}_{\mathrm{PAR}}{ }^{2}\right) / 2 \mathrm{RSD}_{\mathrm{A}} \mathrm{RSD}_{\mathrm{ISTD}}
$$

using the RSD values for the analyte peak (A), the integrated ISTD peak (ISTD), and their ratio (PAR). This coefficient is a measure of the integrated ISTD's correlation with the analyte, in other words, how well it improves the precision of the analyte quantification. Correlation coefficients are calculated for each $\mathrm{NaCl}$ concentration and then are averaged to give a final correlation value for each integrated ISTD (where $\rho=1$ is perfect and $\rho=0$ has no correlation).

When the outlier chip is not used for $\mathrm{Na}$ /“Cs" peak area determination, the average analyte-ISTD correlation is almost identical for all ratios: 0.831 (heights) and 0.856 (areas) for 


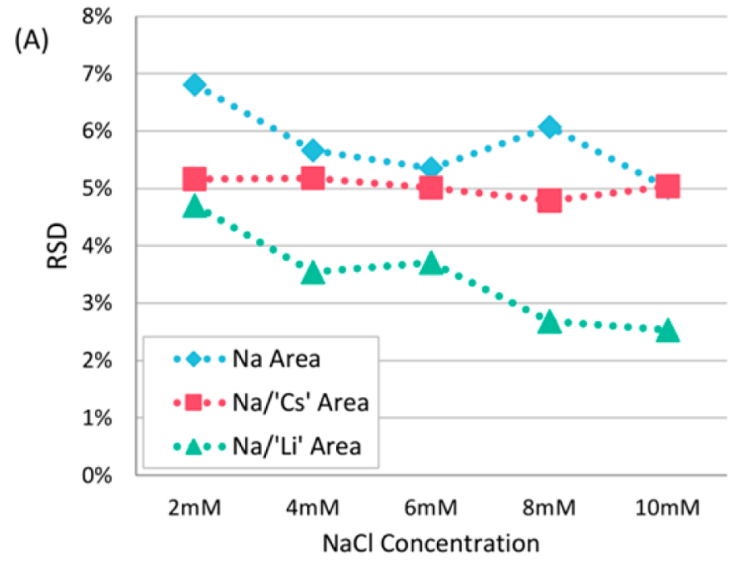

(B)
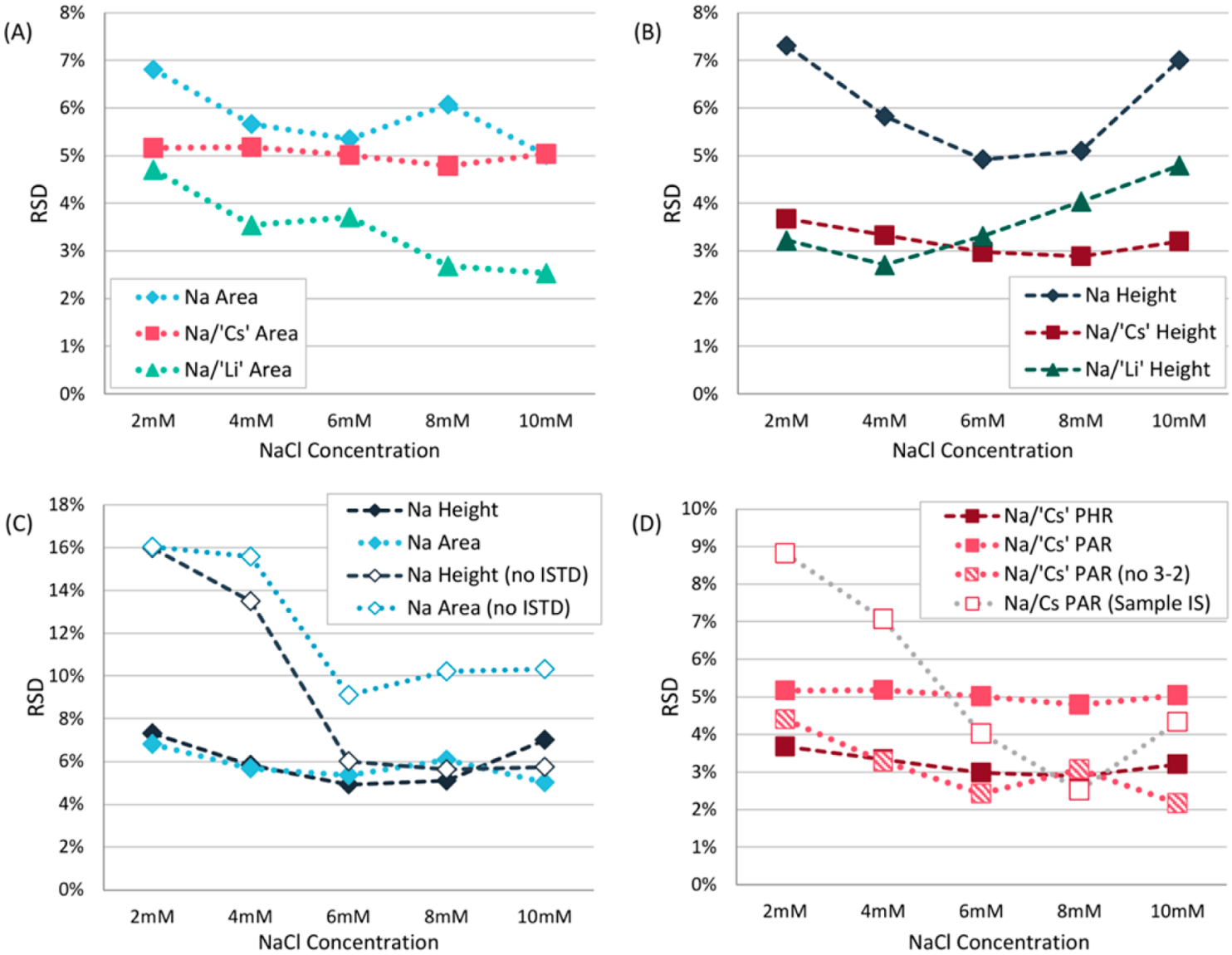

Figure 6. RSDs for $\mathrm{Na}, \mathrm{Na} / “ \mathrm{Cs}$ ”, and $\mathrm{Na}$ /“Li” are compared for peak areas (A) and heights (B). In panel C, Na peak area and height RSDs for each $\mathrm{NaCl}$ concentration are compared to our previous study (ref 10) (no integrated ISTDs in the BGE); the values found in this work are noticeably better, especially at lower concentrations. Panel D compares Cs as both an integrated and traditional ISTD, illustrating that the integrated ISTD values are superior. PHR stands for peak height ratio. RSDs are calculated from six loads per chip, five repeated measurements per one load ( 30 data points per chip, for a total of 180 data points at each concentration level).

$\mathrm{Na} /$ “Li”; 0.856 (heights) and 0.853 (areas) for $\mathrm{Na}$ “"Cs”. Including this outlier chip lowers the $\mathrm{Na} /{ }^{\text {"Cs" }}$ " peak area correlation to 0.729 .

Integrated ISTD versus Traditional ISTD. Figure 6C further compares the Na analyte peak area and height RSDs for this experiment set with the results from the traditional ISTD method. ${ }^{10}$ Note that the actual areas and heights are depicted without any correction by an ISTD. At low $\mathrm{NaCl}$ concentrations $(2-4 \mathrm{mM})$ a 2 -fold increase in precision is seen in the integrated ISTD data. Also of note is the comparable RSDs for both $\mathrm{Na}$ peak areas and heights in the integrated ISTD method, with neither being clearly superior. We attribute the improved (relative to our previous work) chipto-chip analyte performance to a reduction in analyte-glass interactions owing to different chip-to-chip surface chemistries. In the integrated ISTD method, there are a comparable amount of strong electrolyte ions ( $\mathrm{Cs}$ and $\mathrm{Li}$ ) present everywhere in the system, which may reduce the influence of analyte-wall interactions leading to increased variability-particularly at low analyte concentrations.

This improvement can further be seen in the traditional ISTD Na/Cs PAR, illustrated in Figure 6D. RSDs for 2-4 mM of $\mathrm{NaCl}$ are inferior to the values found with the integrated ISTD method, even though they halved the analyte's initial variability. From this figure, we can conclude that the integrated "Cs" ISTD height ratios perform clearly superior to the traditional method, as well as the peak area ratios when the outlier chip is not considered.

Table 1 summarizes the data collected in this paper with the integrated ISTD method and compares it to the traditional method. When considering most parameters, the integrated ISTD values either match or are superior to the traditional method values. The notable exception is the Cs. $\mathrm{Na}$ correlation coefficient $(\rho)$ which is 0.922 for the traditional ISTD and $\sim 0.85$ for the integrated ISTD method. This indicates that the degree with which $\mathrm{Cs}$ improved the $\mathrm{Na}$ precision was better in the traditional method; however, the final precision is more important than the correlation. Here, the integrated "Cs" ISTD is equal or better.

\section{CONCLUSION}

We have shown that the integrated ISTD method is a promising means for improving quantitative precision in disposable microchips, owing to its matched or superior results when compared to the traditional ISTD method. In combination with the fact that this method is suitable for operation by unskilled users, the potential value of the method strongly increases. Furthermore, indication is given that integrated ISTDs may be less bound by substance selection than the traditional method; Cs and $\mathrm{Li}$ performed equally well as integrated ISTDs, while Cs was clearly the better choice in the traditional method. Under these experimental conditions- 
Table 1. Comparison of Integrated ISTD Method and Traditional ISTD Method $^{a}$

\begin{tabular}{|c|c|c|c|}
\hline & \multicolumn{2}{|c|}{ Integrated ISTD } & Traditional \\
\hline Migration Time & \multicolumn{2}{|c|}{ (RSD) } & (RSD) \\
\hline $\mathrm{Na}$ & \multicolumn{2}{|c|}{$2.93 \%$} & $3.60 \%$ \\
\hline $\mathrm{Na} / \mathrm{Cs}$ & \multicolumn{2}{|c|}{$1.06 \%$} & $1.76 \%$ \\
\hline $\mathrm{Na} / \mathrm{Li}$ & \multicolumn{2}{|c|}{$0.98 \%$} & $0.82 \%$ \\
\hline $\mathrm{Na}(\text { new })^{b}$ & \multicolumn{2}{|c|}{$0.71 \%$} & $0.57 \%$ \\
\hline $\begin{array}{l}\text { Calibration Curve } \\
\text { Fitting }\end{array}$ & $\begin{array}{l}\text { Height } \\
\left(x^{2}, R^{2}\right)\end{array}$ & $\begin{array}{c}\text { Area } \\
\left(x, R^{2}\right)\end{array}$ & $\begin{array}{c}\text { Area } \\
\left(x, R^{2}\right)\end{array}$ \\
\hline $\mathrm{Na}$ & 0.970 & 0.984 & 0.943 \\
\hline $\mathrm{Na} / \mathrm{Cs}$ & 0.986 & $\begin{array}{c}0.993 \\
(n=5)\end{array}$ & 0.991 \\
\hline $\mathrm{Na} / \mathrm{Li}$ & 0.988 & 0.997 & 0.971 \\
\hline $\begin{array}{l}\text { ISTD·Analyte } \\
\text { Correlation }\end{array}$ & Height ( $\rho)$ & Area $(\rho)$ & Area ( $\rho)$ \\
\hline $\mathrm{Cs} \cdot \mathrm{Na}$ & 0.856 & $\begin{array}{l}0.853 \\
(n=5)\end{array}$ & 0.922 \\
\hline $\mathrm{Li} \cdot \mathrm{Na}$ & 0.831 & 0.856 & 0.636 \\
\hline Peak Size & $\begin{array}{l}\text { Height } \\
\text { (RSD) }\end{array}$ & $\begin{array}{l}\text { Area } \\
\text { (RSD) }\end{array}$ & $\begin{array}{l}\text { Area } \\
\text { (RSD) }\end{array}$ \\
\hline $\mathrm{Na}$ & $4.9-7.3 \%$ & $5.0-6.8 \%$ & $9.1-16.0 \%$ \\
\hline $\mathrm{Na} / \mathrm{Cs}$ & $2.9-3.7 \%$ & $\begin{array}{c}2.2-4.4 \% \\
(n=5)\end{array}$ & $2.5-8.8 \%$ \\
\hline $\mathrm{Na} / \mathrm{Li}$ & $2.7-4.8 \%$ & $2.5-4.7 \%$ & $6.3-14.2 \%$ \\
\hline
\end{tabular}

${ }^{a}$ Reference $10 .{ }^{b} \mathrm{Na}$ (new) refers to eq 2 .

also owing to the improved analyte peak precision alone-the integrated ISTD method is even superior to the traditional method. Future work will evaluate the integrated ISTD method for more complex and application relevant sample matrixes.

\section{ASSOCIATED CONTENT}

\section{S Supporting Information}

The Supporting Information is available free of charge on the ACS Publications website at DOI: 10.1021/acs.analchem.6b04172.

Image of the symmetric Huber function baseline approximation applied to one electropherogram, simulated $\mathrm{Na}$ ion concentration in the channel after injection plotted vs starting $\mathrm{NaCl}$ concentration in the sample well, visual comparison of peak area RSDs at each $\mathrm{NaCl}$ concentration, tabulated migration time reproducibility data, and tabulated calibration curve data (PDF)

\section{AUTHOR INFORMATION}

\section{Corresponding Author}

*E-mail: j.c.t.eijkel@utwente.nl. Fax: +31-53-489-5653.

\section{Author Contributions}

All authors have given approval to the final version of the manuscript.

\section{Notes}

The authors declare no competing financial interest.

\section{ACKNOWLEDGMENTS}

This work was financially supported by a Spinoza Grant (AvdB) and the Czech Science Foundation Grant No. 15$18424 \mathrm{Y}$

\section{REFERENCES}

(1) Ryvolová, M.; Macka, M.; Preisler, J. TrAC, Trends Anal. Chem. 2010, 29 (4), 339-353.

(2) Breadmore, M. C. J. Chromatogr. A 2012, 1221, 42-55.

(3) Floris, A.; Staal, S.; Lenk, S.; Staijen, E.; Kohlheyer, D.; Eijkel, J.

C. T.; van den Berg, A. Lab Chip 2010, 10 (14), 1799-1806.

(4) Staal, S.; Ungerer, M.; Floris, A.; Ten Brinke, H.-W.; Helmhout, R; Tellegen, M.; Janssen, K.; Karstens, E.; van Arragon, C.; Lenk, S.; Staijen, E.; Bartholomew, J.; Krabbe, H.; Movig, K.; Dubský, P.; van den Berg, A.; Eijkel, J. Electrophoresis 2015, 36 (5), 712-721.

(5) Altria, K. D.; Glaxosmithkline, R. LC-GC Eur. 2002, 15 (9), 588594.

(6) Dose, E. V.; Guiochon, G. A. Anal. Chem. 1991, 63 (11), 11541158.

(7) Blanco-Heras, G. A.; Turnes-Carou, M. I.; López-Mahía, P.; Muniategui-Lorenzo, S.; Prada-Rodríguez, D.; Fernández-Fernández, E. Electrophoresis 2008, 29 (6), 1347-1354.

(8) Revermann, T.; Götz, S.; Künnemeyer, J.; Karst, U. Analyst 2008, 133 (2), 167-174.

(9) Masár, M.; Bomastyk, B.; Bodor, R.; Horčičiak, M.; Danč, L.; Troška, P.; Kuss, H.-M. Microchim. Acta 2012, 177 (3-4), 309-316.

(10) Bidulock, A. C. E.; van den Berg, A.; Eijkel, J. C. T. Electrophoresis 2015, 36 (6), 875-883.

(11) Gaš, B.; Kenndler, E. Electrophoresis 2004, 25 (23-24), 39013912.

(12) Beckers, J. L. J. Chromatogr. A 1994, 679 (1), 153-165.

(13) Štědrý, M.; Jaroš, M.; Gaš, B. J. Chromatogr. A 2002, 960 (1-2), 187-198.

(14) Vrouwe, E. X.; Luttge, R.; van den Berg, A. Electrophoresis 2004, 25 (10-11), 1660-1667.

(15) Mazet, V.; Carteret, C.; Brie, D.; Idier, J.; Humbert, B. Chemom. Intell. Lab. Syst. 2005, 76 (2), 121-133.

(16) Gaš, B.; Hruška, V.; Dittmann, M.; Bek, F.; Witt, K. J. Sep. Sci. 2007, 30 (10), 1435-1445.

(17) Jaroš, M.; Hruška, V.; Štědrý, M.; Zusková, I.; Gaš, B. Electrophoresis 2004, 25 (18-19), 3080-3085.

(18) Hruška, V.; Jaroš, M.; Gaš, B. Electrophoresis 2006, 27 (5-6), 984-991.

(19) Gaš, B. PeakMaster and Simul 5. http://echmet.natur.cuni.cz/ download (accessed Oct 21, 2016).

(20) Štědrý, M.; Jaroš, M.; Včeláková, K.; Gaš, B. Electrophoresis 2003, 24, 536-547.

(21) Hruška, V.; Štědrý, M.; Včeláková, K.; Lokajová, J.; Tesařová, E.; Jaroš, M.; Gaš, B. Electrophoresis 2006, 27 (23), 4610-4617.

(22) Blas, M.; Delaunay, N.; Rocca, J.-L. Electrophoresis 2008, 29 (1), $20-32$.

(23) Vrouwe, E. X.; Luttge, R.; Olthuis, W.; van den Berg, A. Electrophoresis 2005, 26 (15), 3032-3042.

(24) Alarie, J. P.; Jacobson, S. C.; Ramsey, J. M. Electrophoresis 2001, 22 (2), 312-317.

(25) Guidance Document for Single Laboratory Validation of Quantitative Analytical Methods-Guidance Used in Support of Preand-Post-Registration Data Requirements for Plant Protection and Biocidal Products; ENV/JM/MONO, No. 204; OECD: Paris, 2014; Vol. 20, pp $23-25$. 